\title{
The evacuation movement in the corridor corner of passenger ships
}

\author{
Zhou Cheng \\ Maritime Academy \\ Dalian Maritime University \\ Dalian, China
}

\author{
Fu Yuhui \\ Maritime Academy \\ Dalian Maritime University \\ Dalian, China
}

\author{
Wang Yantong \\ Maritime Academy \\ Dalian Maritime University \\ Dalian, China
}

\begin{abstract}
The corner given in IMO MSC.1/Circ.1238 is the main object be analyzed, and the emphasis of this thesis is to analyze the evacuation speed in corner. Based on mechanical analysis, use the methods of sports biomechanics, and consider centrifugal force in non-inertial coordinate system, this thesis educe the range of speed as the ship is upright or tilt in some certain angles.
\end{abstract}

Key Words-Passenger Ship, Inclination, Corner, Evacuation INTRODUCTION

Corner of the corridor is the area that we often have to go through in the process of evacuation, and is critical areas which connected two straight corridors. Straight evacuation corridor suddenly encountered the corner will change evacuees evacuation behavior. The evacuation directions changes, at the same time, the evacuation speed along the corner of vertical and horizontal changes larger.If evacuees stopping power is not enough, that can easily result in fall even trigger a stampede, so the corner of the corridor is extremely important evacuation bottleneck area. It is obviously an extremely important study of the regional impact of evacuees in the evacuation process, improve the efficiency of evacuation of the region can effectively improve the efficiency of the evacuation of the entire evacuation process.

\section{I . CORRIDOR CORNER EXPLODED PARTITION}

The description of the corner in IMO MSC.1/Circ.1238 [1] is as the following Figure shows.

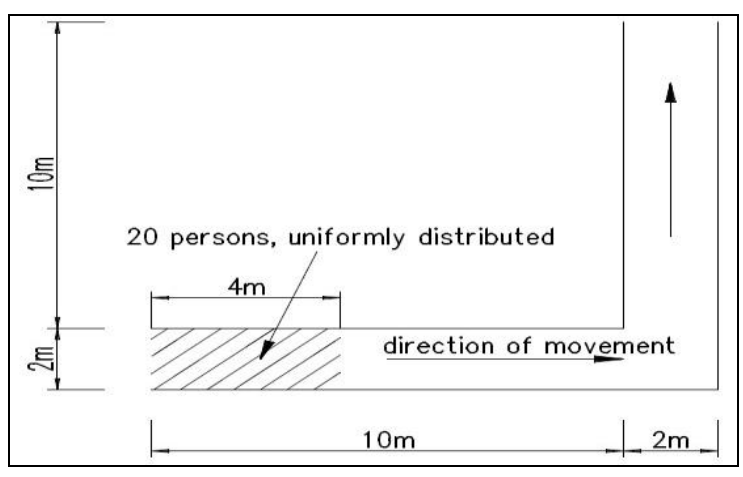

Fig.1 Corner on the passenger ship
The corner is $90^{\circ}$ right angle corner, the width of meters. For the convenience of discussion, in this paper, make the direction of the arrow in the longitudinal corridor as the direction of ahead, the lateral corridor direction of the arrows in the figure as evacuees' direction of movement.

According to the study of Melinek and Booth [2], the personnel walking speed increase, the density decreases at the outside of the curved road, the personnel speed decreases, the density increased at the inside of the curved road, whereby the center line as a reference to separate the angular disposition into the inner and outer sides. Also, because the inclination of the ship's influence on the evacuation, for the convenience of analysis, the corner is divided into upper and lower sides, as the following Figure 2 shows.

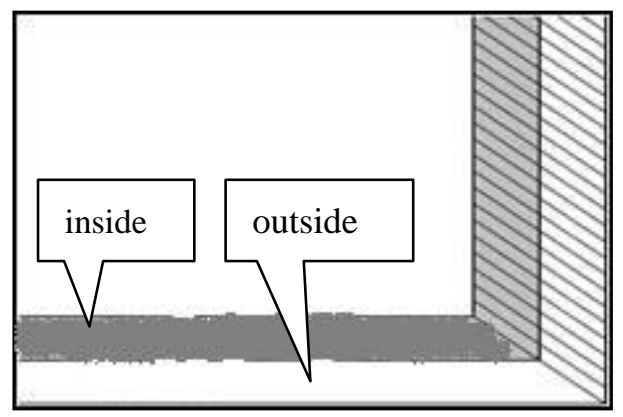

Fig. 2 The 4 sides of corner

Further to determine the studied corner within the range shown in Figure 3, the two solid lines in the figure with the center line, the diagonal together divided the angular disposition into four regions of $\mathrm{A}, \mathrm{B}, \mathrm{C}$ and $\mathrm{D}$.

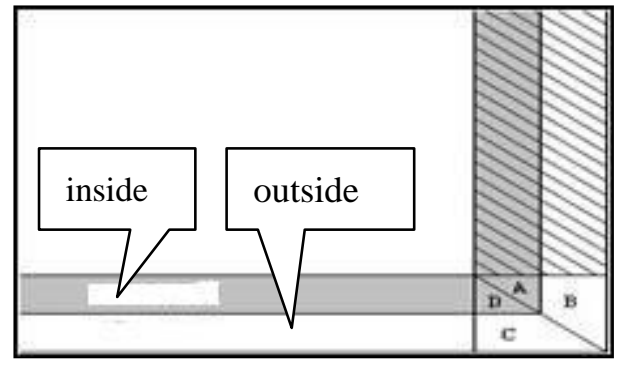

Fig.3 Four fields of corner 


\section{II .DETERMINATION OF THE FRICTION COEFFICIENT} AND THE MAXIMUM INCLINATION ANGLE

Generally the passenger ship corridor will lay some plastic coverings, the common sole material are rubber, plastics, leather, etc. Access to relevant information, the coefficient of friction between some commonly used materials [3] as shown in Table 1 below.

Tab.1 The friction factor of some materials

\begin{tabular}{|c|c|c|}
\hline \multirow{2}{*}{ Material } & \multicolumn{2}{|c|}{ Friction coefficient } \\
\cline { 2 - 3 } & $\begin{array}{c}\text { Static friction } \\
\text { coefficient }\end{array}$ & Friction coefficient \\
\hline Leather - Metal & $0.4 \sim 0.6$ & \\
\hline Rubber - Rubber & 0.5 & $0.03 \sim 0.05$ \\
\hline Leather - Wood & $0.4 \sim 0.5$ & \\
\hline Leather - Black Metal & $0.30 \sim 0.50$ & \multicolumn{2}{|c|}{} \\
\hline Cast Iron -Leather & 0.55 & 0.28 \\
\hline
\end{tabular}

In terms of the comfort of the movement, that the friction coefficient generally between 0.4 and 0.7 is suitable. The common coefficient of friction between the sole and passenger ships corridor floor is also within this range, if there are lubricants on the surface, such as water, the friction coefficient decreases. Taking into account that the smaller the friction coefficient of the evacuees in the movement of the corner is the more prone to slipping, therefore make the coefficient of friction between 0.3 and 0.7 .

The evacuees set a mass M, evacuees shoes friction between the sole and the floor is $\mathrm{f}$, the friction coefficient is $\mu$, the gravitational acceleration $g$ take $9.8 \mathrm{~m} / \mathrm{s} 2$, in the case of non-slip the friction force is: $\mathrm{f}=\mu \mathrm{N}=\mu \mathrm{Mg} \cos \alpha$.

Wherein $\alpha$ is the inclination, friction can be obtained according to the force balance: $f=M g \sin \alpha$.

The above two formulas are equal, we can get $\mu \mathrm{Mg} \cos \alpha=\mu \mathrm{Mg} \sin \alpha$, namely $\tan \alpha=\mu$. According to the foregoing $\mu$ ranges from 0.3 to 0.7 , so we can get the maximum inclination angle ranges from $16.7^{\circ}$ to $35.0^{\circ}$ in the corresponding coefficient of friction. When the angle is $16.7^{\circ}$ evacuees will slip, tilt $35^{\circ}$ easier to slip under the same coefficient of friction. In most of the ship simulation, when the ship's sway angle beyond $30^{\circ}$, we always identify the ship as overturning. Considering all the above factors, we assume the maximum inclination of the passenger ship between $30^{\circ} \sim 35^{\circ}$.

III.THE DYNAMICS ANALYSIS OF EVACUATION AT THE CORNER
When passenger ships need to evacuate passengers in an emergency situation, may be in the upright, tilt state. The personnel evacuation dynamics analysis of passenger ships' corridor corner should also take the different states of passenger ships into account.

\section{A. Upright}

The evacuees under the action of the gravity $\mathrm{Mg}$, the role $\mathrm{N}$, and the friction $f$, friction provide centripetal force. In the corner of the road, the evacuees' body will title toward the inside of the corner. Set human inclination is $\beta$. In rotating non-inertial system, an inertial forces (centrifugal force) need to be introduced to Newton's law, so that evacuees in the rotation non-inertial system is in a state of equilibrium, by the four force of gravity $\mathrm{Mg}$, supportive $\mathrm{N}$, frictional force $\mathrm{f}$, and a centrifugal force $\mathrm{F}$ role, shown in Figure 4, to simplify people into a pole.

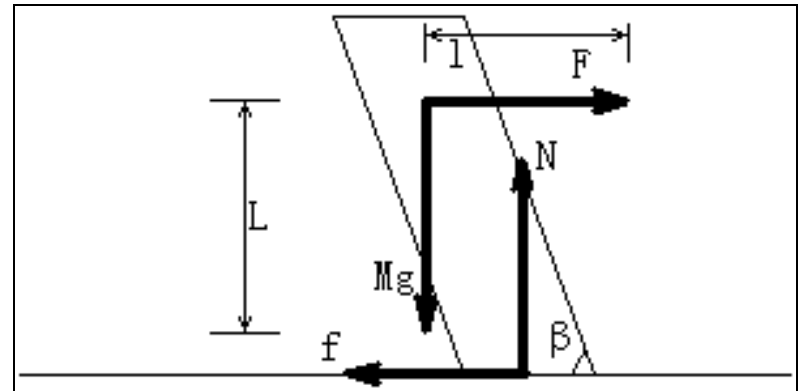

Fig.4 The force-balance of evacuee in corner

The friction force and support force acts on the soles and corridors contact point, gravity and centrifugal force acts on the center of gravity. Two sets of forces are synthesized.

Based on the force rod theory, to make the "pole" (staff) balance the two forces should role in a straight line along the direction of the "pole" and the two forces balance. By the vector relationship between the various forces, the aggregate external force is 0 , to maintain a balance.

Vertical direction: $\mathrm{N}=\mathrm{Mg}$

Horizontal direction: $\mathrm{F}=\mathrm{f}$, that is $M \frac{v^{2}}{R}=\mu N$

Where $\mathrm{R}$ is the evacuation of personnel rotation radius, combine the above two equations we can get $M \frac{v^{2}}{R}=\mu M g$, that is $\frac{v^{2}}{R}=\mu g$.

The total torque is 0 , that is $F \times L=G \times l, \mathrm{G}=\mathrm{Mg}$ is evacuees suffered gravity.

Also, because the movement of the people is extremely 
complex, it is not easy to determine the turning radius of the corner. Now assume that an evacuation route in the corner is a quarter of arc, radius is $1 \mathrm{~m}$. And most of the evacuees move from the zone D to Zone B.

Substitute the radius of curvature $R=1(\mathrm{~m})$ into the formula (3), make the coefficient of friction 0.3 we can get the speed $v=$ $1.71 \mathrm{~m} / \mathrm{s}$. According to IMO MSC.1/Circ.1238, the biggest evacuation speeds of young men and women are $1.85 \mathrm{~m} / \mathrm{s}$ and $1.55 \mathrm{~m} / \mathrm{s}$, what can basically meet the requirements of the speed limit. When the friction coefficient take 0.7 , the speed $v=2.62 \mathrm{~m}$ / s. According to IMO MSC.1/Circ.1238, the speed can meet the limits.

\section{B. Leftist}

When the passenger ship tilts to the left, evacuees first go uphill along the horizontal walkway connecting corridor corner, and then move forward in the vertical left aisle, at the same time, the overall do turning motion around the corner. Evacuees in the corner move forward along the walkway uphill in the first half of the walk, and continue to move along the inclined walkway at the rear half away of the corner.

Compare the study about the change of evacuation speed with the change of inclination when the corridor tilting back and forth and with the left and right tilt angle, based on the study of the ETH research institutions, we can find that the passenger ship tilt $0^{\circ} \sim 8^{\circ}$ in the front and rear direction makes little difference on the evacuees' walking speed. The difference became significant when the slope beyond $8^{\circ}$. When the ship tilt $0^{\circ} \sim 15^{\circ}$ in the left and right direction, speed changes a little, the change became significant when the angle beyond $15^{\circ}$. Therefore, this study focuses on the inclined slope from $8^{\circ}$ to $35^{\circ}$.

Evacuees make curvilinear motion around the corner, so we can treat it as a circular motion around the corner. Use the same analysis methods like upright, we need to introduce a centrifugal force in the non-inertial system to use Newton's laws. By the vector relationship between the various forces, the aggregate external force is 0 , to maintain a balance, that is

The vertical direction, $M g=N \cos \phi-f \sin \phi$

The horizontal direction, $F=f \cos \phi+N \sin \phi$

Where the size of the frictional force $\mathrm{f}$ is $f=\mu N$, supportive $N=M g \cos \phi+F \sin \phi$, substitute into the above formula we can get: $F=\mu N \cos \phi+N \sin \phi$ $=M g\left(\mu \cos ^{2} \phi+\sin \phi \cos \phi\right)+F\left(\mu \sin \phi \cos \phi+\sin ^{2} \phi\right)$
That is, $F=\frac{M g\left(\mu \cos ^{2} \phi+\sin \phi \cos \phi\right)}{1-\mu \sin \phi \cos \phi-\sin ^{2} \phi}$

Also $\mathrm{F}$ is the centrifugal force, so $F=M \frac{v^{2}}{R}$

The two equations (6), (7) are equal, so we can get:

$$
v^{2}=\frac{R g\left(\mu \cos ^{2} \phi+\sin \phi \cos \phi\right)}{1-\mu \sin \phi \cos \phi-\sin ^{2} \phi}
$$

$\mathrm{R}$ is evacuees turning radius, we take $1 \mathrm{~m}$ when the ship at upright position, and we take $R=\cos \phi \quad(\mathrm{m})$ when the ship tilt left. Substitute R into the formula (8), we can get:

$$
\begin{aligned}
v^{2}= & \frac{g \cos \phi\left(\mu \cos ^{2} \phi+\sin \phi \cos \phi\right)}{1-\mu \sin \phi \cos \phi-\sin ^{2} \phi} \\
= & \frac{g \mu \cos ^{3} \phi+g \sin \phi \cos ^{2} \phi}{1-\mu \sin \phi \cos \phi-\sin ^{2} \phi}
\end{aligned}
$$

The friction coefficient $\mu$ values in the range 0.3 to $0.7, \varphi$ values in the range $8^{\circ} \sim 35^{\circ}$. Thereby substitute the two number into the above formula we can get evacuation velocity $\mathrm{v}$ is $2.11 \mathrm{~m} / \mathrm{s} \leq \mathrm{v} 1 \leq 4.69 \mathrm{~m} / \mathrm{s}$. We can find that the turning speed when the ship tilt left is faster than the speed when the ship upright. That is to say when the ship tilt left is conducive to evacuation in a sense.

\section{Rightist}

When the passenger ship tilts right, evacuees first go downhill along the first half of the transverse at the corner, and then continue to move forward along the latter half of the right-leaning longitudinal aisle. Meanwhile the evacuees do the turning movement in the entire corner. Evacuees make curvilinear motion around the corner, so we can treat it as a circular motion around the corner. We need to introduce a centrifugal force in the non-inertial system to use Newton's laws. By the vector relationship between the various forces, the aggregate external force is 0 , to maintain a balance, that is, In the vertical direction, $M g=N \cos \phi+f \sin \phi(10)$

In the horizontal direction, $F=f \cos \phi-N \sin \phi$

Submit $f=\mu N$ and $N=M g \cos \phi-F \sin \phi$ into (11) yields:

$$
\begin{aligned}
& F=\mu N \cos \phi-N \sin \phi \\
& =(M g \cos \phi-F \sin \phi)(\mu \cos \phi-\sin \phi) \\
& =M g\left(\mu \cos ^{2} \phi-\sin \phi \cos \phi\right)-F\left(\mu \sin \phi \cos \phi-\sin ^{2} \phi\right)
\end{aligned}
$$

That is $F=\frac{M g\left(\mu \cos ^{2} \phi-\sin \phi \cos \phi\right)}{1+\mu \sin \phi \cos \phi-\sin ^{2} \phi}$, And $\mathrm{F}$ is the 
centrifugal force, thereby $F=M \frac{v^{2}}{R}$, These two simultaneous

can get: $v^{2}=\frac{R g\left(\mu \cos ^{2} \phi-\sin \phi \cos \phi\right)}{1+\mu \sin \phi \cos \phi-\sin ^{2} \phi}$

The friction coefficient $\mu$ values in the range 0.3 to $0.7, \varphi$ values in the range $8^{\circ} \sim 35^{\circ}$. Thereby substitute the two number into the above formula we can get evacuation velocity $\mathrm{v}$ is $0 \mathrm{~m} / \mathrm{s}$ $\leq \mathrm{v} \leq 2.22 \mathrm{~m} / \mathrm{s}$. The evacuation speed limit of $2.22 \mathrm{~m} / \mathrm{s}$ is the inclination to take minimum $8^{\circ}$ and friction coefficient whichever is greater 0.7 . By the previously described shows that the impact on evacuation is not obvious when passenger ships' corridors tilt $0^{\circ} \sim 15^{\circ}$ in the horizontal direction and tilt $0^{\circ}$ to $8^{\circ}$ in the front-rear direction, and take into account of the error of the coefficient of friction, and the turning radius. So the evacuation speed maximum of $2.22 \mathrm{~m} / \mathrm{s}$ is reasonable. Figure 5 is the three dimensional figure depicted by Matlab about the evacuation speed changes with the change of the angle $\varphi$ and the friction coefficient $\mu$.

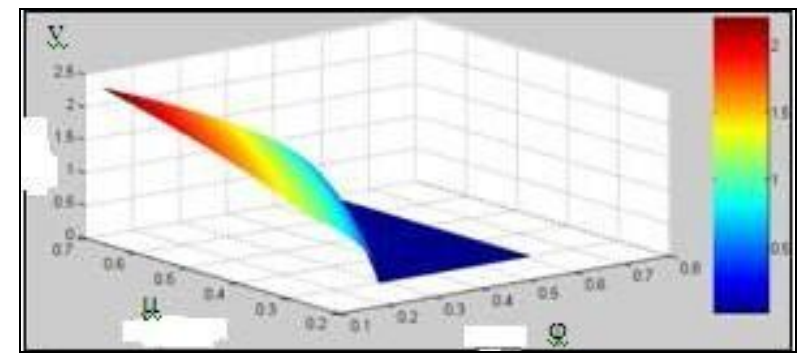

Fig.5 Evacuation speed as a function of obliquity and friction factor when leaning right

From Figure 5 (b) we can see the inclination angle and the friction coefficient values in a certain area, the evacuation speed $\mathrm{v}$ is 0.It means that the inclination angle and the friction coefficient is not sufficient to ensure the evacuation of personnel to be evacuated to a certain speed, evacuees will slip.

D. Trim

Evacuation of the trim is similar to the evacuation of the heeling, can refer to the method of the heeling. Of course, the pitch angle is always small, and its impact is not very significant.

\section{IV.PROPOSALS}

The most critical of the evacuation at the corner is to prevent persons slipping in the meanwhile to ensure evacuation speed. From the above analysis, we can see that the corner of the corridor tilt to the inside when the ship left-leaning, so the gravity and friction jointly provide the centripetal force, which was needed when the staff turn, thus it is conducive to prevent the staff from slipping at a faster speed. When passenger ship tilt right, the evacuees' gravity contributes to offset part of the friction, so the centripetal force that fiction can provide decreases. For the possible scenarios of the evacuation around the corner, and combine the conclusion I get from this paper, I come up with the following proposals.

\section{A. Attention to the small details of the evacuation}

For example, add carpet to increase the coefficient of friction, the layout at the corner should be as brief as possible, some unnecessary bonsai and other furnishings should not be placed at the corner. In addition, the convex corners at the right-angle corner tend to be sharp, should be smooth handling.

\section{B. Crew}

When there is an emergency need to evacuate passengers, should deploy more staff to guide evacuees to choose a more suitable path for evacuation, to divert passengers according to different tilt directions of the passenger ships, to allow more people to favor evacuation around the corner.

\section{Passengers}

There are less requirements of the stop force at the outside of the corner. So the vulnerable groups should try to go along the outside of the corner. At the same time, most people will choose to evacuate from the Area D to Area B, this evacuation route can avoid conflict with the majority of evacuees.

D. Improve the evacuation channel design

Develop the right-angle corner into a curved corner, curved corner can effectively improve the efficiency of evacuation at the corner, what has been confirmed at the road traffic. I believe that similar improvements can be achieved very good results in the corner of the passenger ship corridor.

\section{REFERENCES}

[1] Guidelines for evacuation analysis for new and existing passenger ships. IMO MSC.1/Circ.1238，30 October， 2007.

[2] S J Melinek, S Booth. The analysis of evacuation time and the movement of crowds in buildings. Building Research Establishment, 1975.

[3] Xu Hao. Mechanical design handbook. Machinery industry press.1991.

[4] Zhang Peihong, Lu Tao, Chen Baozhi, Observation and analysis of pedestrian flow under time pressure ergonomics,

[5] Huang Qiyou. Research on underground evacuation model bases on crowd's pedestrian flow.2008 